Supporting Information

\title{
Protease degradation of protein coronas and its impact on cancer cells and drug payload release
}

Cristina Rodriguez-Quijada ${ }^{1}$,Helena de Puig ${ }^{4}$, Maria Sánchez-Purràl ${ }^{l}$,Chandra Yelleswarapu ${ }^{2}$,

Jason J. Evans ${ }^{3}$, Jonathan P. Celli ${ }^{2}$, Kimberly Hamad-Schifferli ${ }^{1,4}$

${ }^{1}$ Department of Engineering, ${ }^{2}$ Department of Physics, and the ${ }^{3}$ Department of Chemistry.

University of Massachusetts Boston, Boston MA 02125

${ }^{4}$ Department of Mechanical Engineering, Massachusetts Institute of Technology, Cambridge, MA 02139

*Corresponding author: Kim.Hamad@umb.edu 


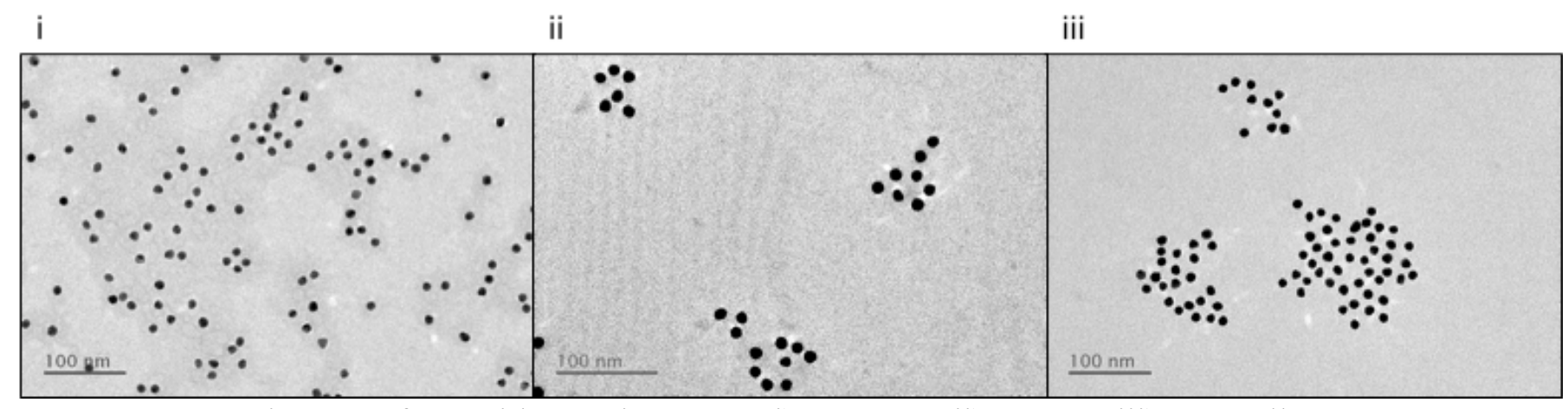

Figure S1. TEM images of NP with protein corona: i) NP-HSA, ii) NP-HS, iii) NP-Coll

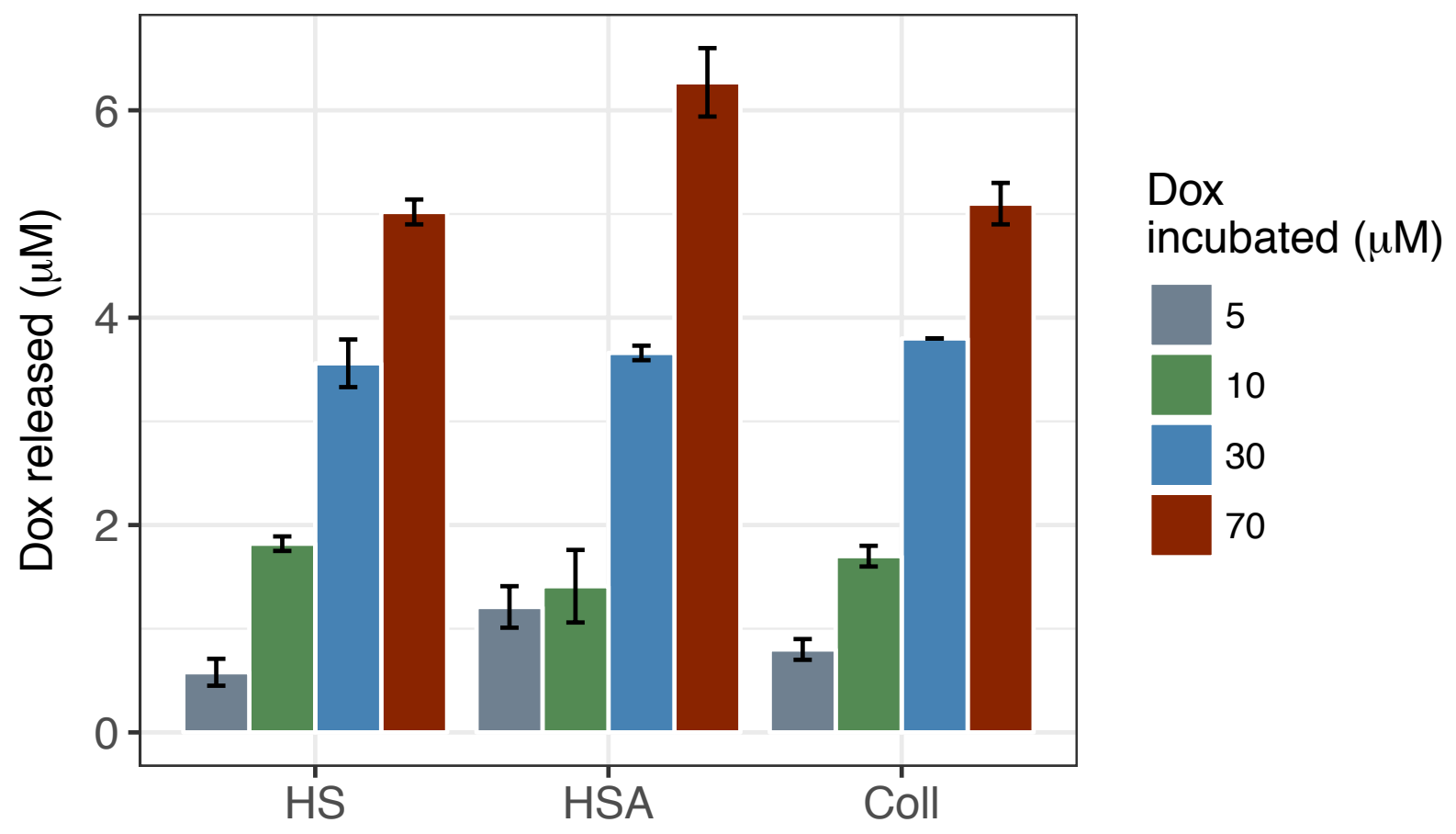

Figure S2: Drug Release after $2 \mathrm{~h}$ of incubation for AuNP-HS, AuNP-HSA, and AuNP-Coll as a function of Dox payload concentration $5 \mu \mathrm{M}$ (gray), $10 \mu \mathrm{M}$ (green), $30 \mu \mathrm{M}$ (blue) and $70 \mu \mathrm{M}$ (red). Error bars represent standard deviation of three replicates. 


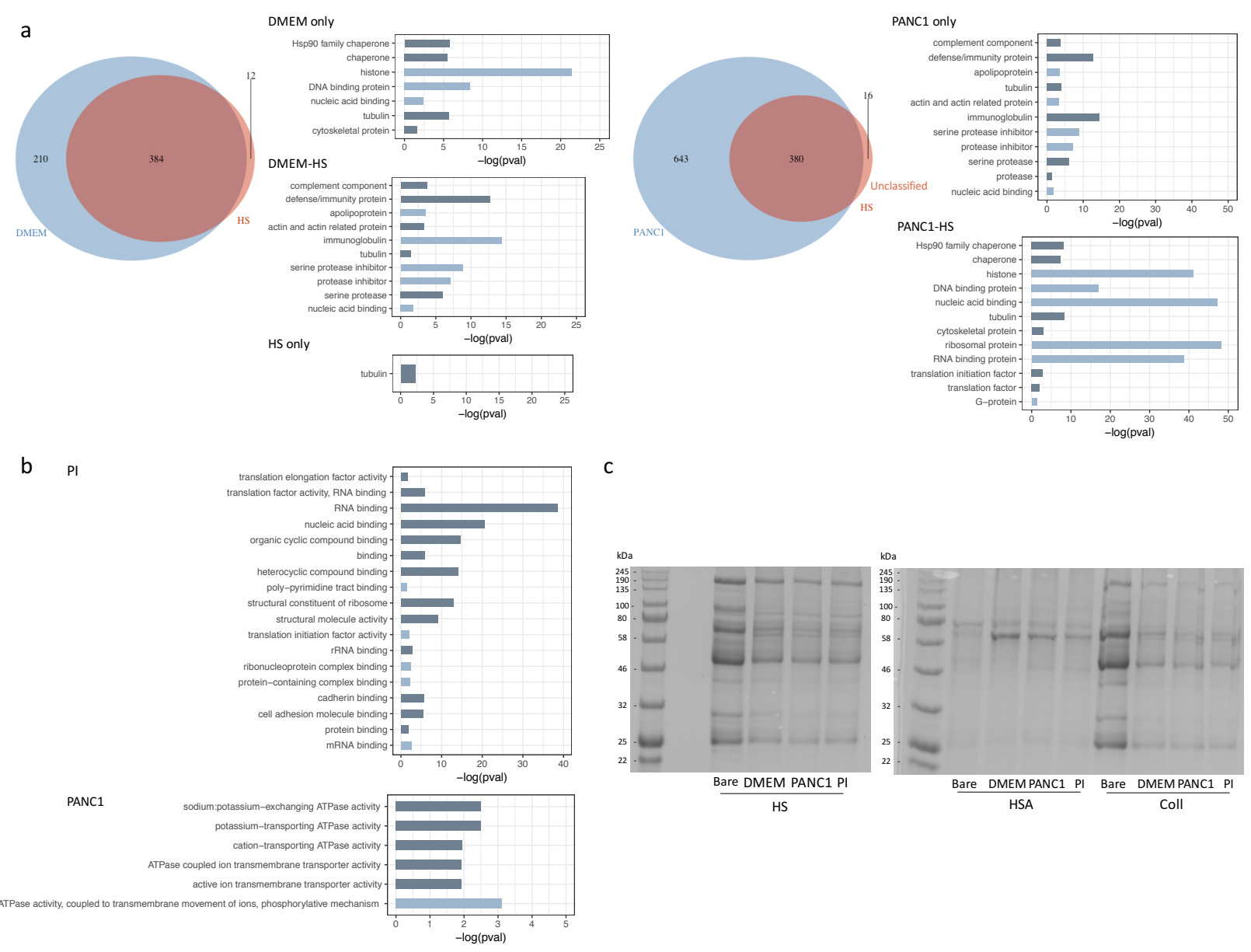

Figure S3. a) Individual Venn Diagrams between DMEM-HS and PANC1-HS with the corresponding PANTHER analysis on unique and shared proteins show a different enrichment of proteins after protein corona exchange and degradation. b) GO molecular function of proteins significantly different in the PC exposed to protease inhibitor (PI) (upper figure) and not exposed to PI (lower figure). GO analysis of biological functions of the protein corona exposed to the proteolytic environment with and without protease inhibitor leads to a $\mathrm{PC}$ with different function. This is indicative of the importance of studying the protein corona in a specific microenvironment to better predict its biological ID. c) SDS PAGE of HS, HSA and Coll systems. 


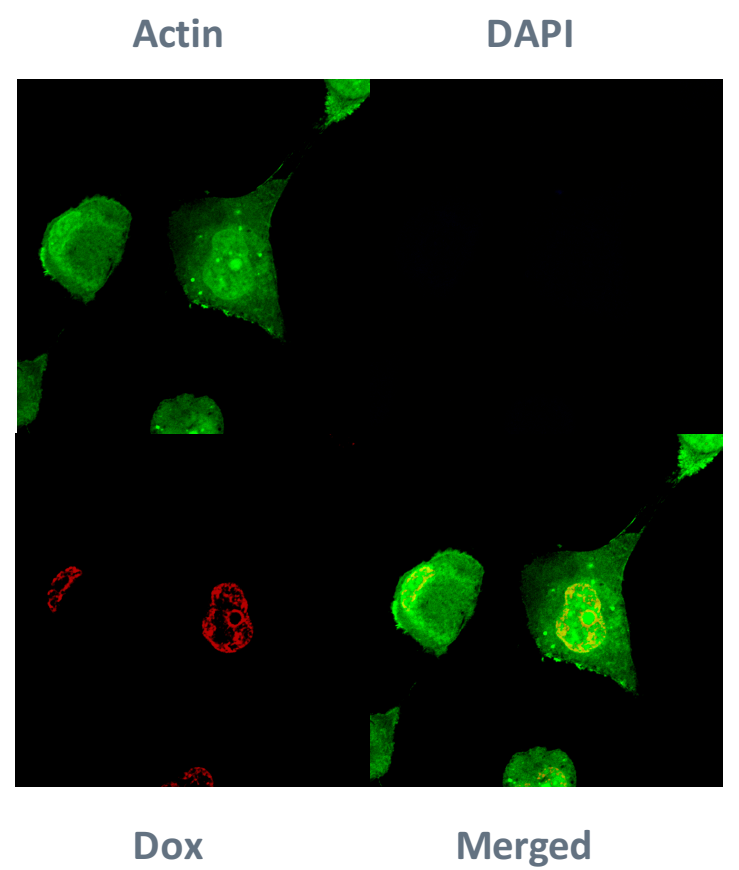

Figure S4: Confocal microscopy of PANC-1 cells incubated with free Dox without DAPI staining. Actin shown in green and doxorubicin in red. 
Table S1: Polydispersity index (PDI) of DLS measurements of bare AuNPs and protein corona complexes.

\begin{tabular}{|l|l|l|c|}
\hline \multicolumn{1}{|c|}{ Sample } & \multicolumn{1}{c|}{ PDI } & \multicolumn{1}{|c|}{ Sample } & PDI \\
\hline \hline AuNP & $0.20 \pm 0.00$ & AuNP-HSA MMP-9 280 24h & $0.34 \pm 0.06$ \\
\hline AuNP-Coll & $0.25 \pm 0.02$ & AuNP-HSA MMP-9 420 24h & $0.31 \pm 0.01$ \\
\hline AuNP-HS & $0.14 \pm 0.02$ & AuNP-HSA MMP-9 560 24h & $0.25 \pm 0.04$ \\
\hline AuNP-HSA & $0.19 \pm 0.02$ & AuNP-Coll MMP-9 280 1h & $0.26 \pm 0.00$ \\
\hline AuNP-Coll +DMEM & $0.34 \pm 0.03$ & AuNP-Coll MMP-9 420 1h & $0.29 \pm 0.02$ \\
\hline AuNP-HS +DMEM & $0.25 \pm 0.03$ & AuNP-Coll MMP-9 560 1h & $0.32 \pm 0.04$ \\
\hline AuNP-HSA +DMEM & $0.34 \pm 0.01$ & AuNP-Coll MMP-9 280 24h & $0.27 \pm 0.01$ \\
\hline AuNP-Coll +MMP-9 active & $0.39 \pm 0.05$ & AuNP-Coll MMP-9 420 24h & $0.27 \pm 0.01$ \\
\hline AuNP-Coll +MMP-9 inactive & $0.35 \pm 0.00$ & AuNP-Coll MMP-9 560 24h & $0.32 \pm 0.04$ \\
\hline AuNP-HS +MMP-9 active & $0.22 \pm 0.01$ & AuNP-HS PANC1 0.5h & $0.18 \pm 0.02$ \\
\hline AuNP-HS +MMP-9 inactive & $0.20 \pm 0.03$ & AuNP-HS PANC1 1h & $0.17 \pm 0.02$ \\
\hline AuNP-HSA +MMP-9 active & $0.71 \pm 0.06$ & AuNP-HS PANC1 2h & $0.19 \pm 0.02$ \\
\hline AuNP-HSA +MMP-9 inactive & $0.35 \pm 0.04$ & AuNP-HS PANC1 24h & $0.18 \pm 0.01$ \\
\hline AuNP-HS MMP-9 280 1h & $0.26 \pm 0.01$ & AuNP-HSA PANC1 $0.5 \mathrm{~h}$ & $0.22 \pm 0.02$ \\
\hline AuNP-HS MMP-9 420 1h & $0.25 \pm 0.01$ & AuNP-HSA PANC1 1 h & $0.20 \pm 0.01$ \\
\hline AuNP-HS MMP-9 560 1h & $0.27 \pm 0.02$ & AuNP-HSA PANC1 2h & $0.22 \pm 0.04$ \\
\hline AuNP-HS MMP-9 280 24h & $0.27 \pm 0.02$ & AuNP-HSA PANC1 24h & $0.22 \pm 0.02$ \\
\hline AuNP-HS MMP-9 420 24h & $0.21 \pm 0.02$ & AuNP-Coll PANC1 2h & $0.24 \pm 0.02$ \\
\hline AuNP-HS MMP-9 560 24h & $0.25 \pm 0.01$ & AuNP-Coll PANC1 4h & $0.26 \pm 0.01$ \\
\hline AuNP-HSA MMP-9 280 1h & $0.33 \pm 0.00$ & AuNP-Coll PANC1 6h & $0.23 \pm 0.01$ \\
\hline AuNP-HSA MMP-9 420 1h & $0.28 \pm 0.02$ & AuNP-Coll PANC1 24h & $0.20 \pm 0.02$ \\
\hline AuNP-HSA MMP-9 560 1h & $0.26 \pm 0.02$ & & \\
\hline
\end{tabular}

